doi: https://doi.org/ 10.5377/paradigma.v28i46.12837

\title{
Vitrinas sobre el Conocimiento de los Cuerpos: El Caso del VIII Congreso Panamericano de Educación Física, México 1982
}

\section{Showcases on the Knowledge of Bodies: The case of the VIII Pan American Congress of Physical Education, Mexico 1982}

Pablo Ariel Scharagrodsky ${ }^{l}$

\begin{abstract}
Resumen
El siguiente trabajo analiza, a partir de una perspectiva socio-histórica con énfasis en la historia social de los saberes y expertos, el VIII Congreso Panamericano de Educación Física realizado en México en 1982. El objetivo central es indagar los saberes transmitidos, la función de los expertos y el papel de las instituciones en el congreso. Entre las conclusiones se identifican continuidades y discontinuidades con relación a otros panamericanos. Entre las continuidades se destacan el rechazo al ‘campeonismo deportivo' y la necesidad de distinguir deporte de educación física. Entre las discontinuidades sobresale un nuevo discurso sobre el deporte y la igualdad de género. En la circulación de estos discursos, los expertos y las instituciones internacionales tuvieron un papel fundamental.

Palabras claves: congreso, panamericanos, educación física, conocimientos, expertos, México
\end{abstract}

\begin{abstract}
The following work analyzes, from a socio-historical perspective with emphasis on the social history of knowledge and experts, the VIII Pan American Congress of Physical Education held in Mexico in 1982. The main objective is to investigate the knowledge transmitted, the function of the experts and the role of institutions in the congress. Among the continuities, the rejection of 'sports championship' and the need to distinguish sport from physical education stand out. Among the discontinuities, a new discourse on sport and gender equality is noted. In the circulation of these discourses, experts and international institutions played a fundamental role.
\end{abstract}

Keywords: congress, physical education, pan american, knowledge, experts, Mexico

${ }^{1}$ pas@unq.edu.ar. Universidad Nacional de Quilmes, Bernal, Argentina. Universidad Nacional de La Plata, La Plata, Buenos Aires, Argentina. https://orcid.org/0000-0001-6305-2017

Recibido 27 de agosto de 2021 / Aceptado 5 de noviembre de 2021 


\section{Introducción}

En 1982 se realizó el VIII Congreso Panamericano de Educación Física en la capital mexicana. El evento fue el resultado de casi cuatro décadas de vínculos e intercambios entre especialistas dedicados a la educación física, los deportes y demás propuestas relacionados con el universo de la cultura física. Este último concepto es entendido como una forma especializada del discurso corporal vinculado con la creación de significados, centrados en las prácticas corporales, que constituyen actividades organizadas e institucionalizadas como el deporte, el ejercicio físico y el ocio activo (Kirk, 2010).

La red de sociabilidad de los congresos panamericanos se inició a principios de los años '40 con el Primer Congreso Panamericano de Educación Física organizado en Brasil en 1943. Al mismo le sucedieron otros seis eventos internacionales realizados en 1946, 1950, 1965, 1970, 1976 y 1980. En todos ellos la participación y el grado de poder e influencia de los delegados y representantes mexicanos fue muy significativo. De hecho México se convirtió en el primer país en organizar el evento en 1982 por segunda vez. Ya lo había hecho con gran éxito en términos de países participantes, delegados y expertos presentes e impacto educativo en el año 1946 en el distrito federal.

Importantes expertos mexicanos circularon por los primeros congresos panamericanos consolidando su autoridad y poder dentro de la disciplina. Por ejemplo, en el Primer Congreso Panamericano de Educación Física organizado en Brasil en 1943, entre los delegados de los trece países participantes, México estuvo presente con el Capitán Miguel Torres Contreras, Pedro Ascencio Rosales y el General Manuel Reyes Iduñate - vicepresidente del congreso en Brasil-, quien siendo Director Nacional de Educación Física y Enseñanza Pre-Militar, posibilitó la concreción del panamericano en su país tres años después. Su participación fue central ya que no solo ofreció un discurso de apertura, sino que presentó cuatro ponencias: "Legislación Nacional sobre terrenos destinados a la recreación organizada", "Aspecto Social y Cultural de la Educación Física", "Las competencias escolares en masa como medio para combatir el 'Campionismo', elevar el coeficiente físico colectivo y crear una conducta deportiva satisfactoriamente eficiente" y "El Deporte como Medio de Fomento de la Espiritualidad, subordinación Consciente y Alejamiento Sectarista de las Juventudes Panamericanas" (Anais Primero Congreso Panamericano de Educação Física Volume I, pp. 52-53, 59-65).

El Segundo Congreso Panamericano de Educación Física fue organizado por la Dirección Nacional de Educación Física y Enseñanza Premilitar, bajo regulación de la Secretaría de la Defensa 
Nacional de México. El evento fue presidido por una de las máximas autoridades militares: el General Antonio Gómez Velasco y se convirtió en un éxito transnacional ya que contó con la participación de delegados y asistentes de diecinueve países de América: Argentina, Brasil, Chile, Costa Rica, Colombia, Cuba, Ecuador, El Salvador, Estados Unidos, Guatemala, Honduras, Nicaragua, Panamá, Paraguay, Perú, Puerto Rico, Uruguay, Venezuela y México (Memoria oficial del II Congreso Panamericano de Educación Física, 1946, p. 343). En la inauguración del congreso estuvieron presentes importantes autoridades militares y educativas (General Francisco Urquiza, General Manuel Ávila Camacho, José Vasconcelos, etc.), diplomáticos y, muy especialmente, docentes de diversos niveles educativos y de la especialidad (Scharagrodsky, 2021a).

A partir del Tercer Congreso Panamericano de Educación Física realizado en Montevideo en 1950 hubo poca participación mexicana en los eventos internacionales. Por ejemplo, en Uruguay hubo un solo representante mexicano: Bernardo Reyes (Informe del III Congreso Panamericano de Educación Física, Delegados Asistentes, 1950, p. 3). También hubo un solo representante mexicano en el Cuarto Congreso Panamericano de Educación Física realizado en Bogotá en 1965: David Ferriz quien expuso sobre "la gimnasia psico-física del eminente sabio francés Dr. Serge Raynaud de la Ferriere" (David Ferriz, Documento de Trabajo No 24, Memorias del IV Congreso Panamericano de Educación Física, 1965). En el Quinto Congreso Panamericano de Educación Física realizado en Buenos Aires en 1970 no figuran mexicanos inscriptos, sin embargo, en las memorias se menciona la ponencia enviada por Celso Enríquez (México) denominada "en defensa de la profesión” (Memorias del V Congreso Panamericano de Educación Física, 1971, pp. 258, 117).

En el Sexto Congreso Panamericano de Educación Física realizado en Carabobo, Venezuela en 1976 estuvieron presentes cuatro delegados mexicanos, entre ellos el profesor Abraham Ferreiro Toledano (Director de Educación Física del Distrito Federal de la ciudad de México) (Acta final del VI Congreso Panamericano de Educación Física y II Gimnasiada Americana, Acta de la Reunión preparatoria, Tomo I, 1976, pp. 2-3). Su papel y autoridad fue central en la constitución de la educación física mexicana moderna. En el Séptimo Congreso Panamericano de Educación Física realizado en la República Dominicana en 1980 estuvieron presentes, entre otros, nuevamente Abraham Ferreiro Toledano y José Jaime Velázquez de México. El primero fue uno de los catorce vice-presidentes electos del evento (Memoria oficial del VII Congreso Panamericano de Educación Física, 1980, p. 5) y fue clave para organizar el congreso panamericano en la capital mexicana en 1982. 
En este contexto, existiendo un campo panamericano de Educación Física consolidado a principios de los años ' 80 , el siguiente trabajo examina y analiza, a partir de una historia social, política y cultural de la educación, la dinámica desarrollada en el VIII Congreso Panamericano de Educación Física realizado en la capital mexicana. Para ello centra el análisis en la presentación y circulación de saberes (Linhales et al., 2019) y los debates generados en dicho espacio, así como quienes fueron los y las participantes, sus inserciones institucionales, sus trayectorias formativas y laborales y las instituciones presentes en dicho espacio.

\section{Discusión Teórica}

Se parte del supuesto de que la educación física como disciplina educativa ha sido un producto moderno vinculado con la gestión, administración, regulación y control de los cuerpos en movimiento, en el marco de los procesos de escolarización y consolidación de los estados educadores modernos. La educación física se consolidó en el 'largo siglo XIX’2 europeo (Vigarello \& Holt, 2005) y fue transmitida, traducida y resignificada en gran parte de América latina. En particular se destacaron cuatro grandes prácticas corporales modernas experimentadas en buena parte de occidente: los deportes, los distintos tipos de gimnasia, ciertos bailes y danzas folklóricas y las actividades recreativas. Todas estas prácticas estuvieron potenciadas en las instituciones educativas modernas por una serie de preocupaciones decimonónicas vinculadas con

La compleja expansión de la vida en algunas ciudades europeas y americanas; la imposición paulatina de nuevos códigos de moralidad y civilidad modernos (hábitos de disciplina, de autocontrol, de competencia, de progreso, etc.); la lucha desde ciertos sectores sociales contra los 'excesos' y los 'peligros' de la creciente vida urbana como el alcoholismo, el sedentarismo, la 'degeneración', el tabaquismo o los 'vicios' sexuales; los incipientes cambios en los procesos de industrialización y sus consecuencias 'no deseadas' en el 'stock físico' de las naciones; la emergencia de los estados nacionales y la contribución en la construcción de un 'canon' físico y de un sentido de pertenencia a la 'comunidad imaginada'; la necesidad de regenerar cuerpos y poblaciones; la producción de cuerpos sanos y aptos para el trabajo y la vida social; la inclusión jerárquica de los grupos subalternos en un nuevo orden corporal, emocional y sensorial; la lucha contra ciertas enfermedades y la promoción de un determinado estilo de vida higiénico y moralmente saludable. (Scharagrodsky, 2011, p. 16)

${ }^{1}$ El 'largo siglo XIX' (The long nineteenth century) fue un término acuñado y utilizado por el reconocido historiador inglés Eric Hobsbawn para referirse al período histórico de 125 años comprendido entre la Revolución francesa (1789) y la Primera Guerra Mundial (1914). 
Estos y otros factores socio-políticos lentamente potenciaron el consumo y la práctica de los deportes, la educación física, las gimnasias y las actividades recreativas dentro y fuera de las instituciones educativas modernas.

Los diferentes países de América latina, cada uno con sus tradiciones y matices locales, desde mediados del siglo XIX participaron activamente en el complejo proceso de globalización, circulación, intercambio, apropiación, imposición y re-significación de dichas prácticas corporales (Linhales et al., 2019). Uno de los espacios donde se discutieron sus beneficios pedagógicos y biomédicos y se propusieron nuevas categorías teóricas con el fin de mejorar las prácticas e imponer ciertos regímenes de verdad sobre la disciplina, fueron los congresos internacionales. Durante buena parte del siglo XX, los congresos vinculados con la educación física, la medicina deportiva o la higiene física condensaron la emergencia de tres dimensiones centrales y constitutivas de la disciplina: los saberes necesarios a la hora de construir la disciplina (tópicos centrales, finalidades, metodologías, teorías sobre la enseñanza y el aprendizaje, etc.), los expertos con autoridad para imponer ciertos regímenes de verdad sobre la disciplina educativa y las instituciones que encuadraron y legitimaron los procesos de intervención sobre los cuerpos en movimiento.

Como se analizó, el VIII Congreso Panamericano de Educación Física realizado en 1982 en México fue un espacio material y simbólico en el cual circularon ciertos saberes, emergieron determinados expertos que se arrogaron la autoridad epistémica de la disciplina y se consolidaron ciertas instituciones internacionales vinculados con el heterogéneo campo de la cultura física moderna. En este punto, conceptualizamos al VIII Congreso Panamericano como una ventana hermenéutica donde es posible identificar los tópicos discutidos -y consensuados- por una comunidad de especialistas, los referentes disciplinares constructores de sentidos y las instituciones que encuadraron y legitimaron determinadas políticas sobre el universo deportivo, recreativo o gímnico moderno. Analizar dichas dimensiones nos permitió comprender, en un tiempo y espacio específico, las lógicas pedagógico-políticas predominantes de la educación física como disciplina educativa, sus continuidades y rupturas de sentidos.

\section{Metodología}

El encuadre metodológico del artículo se sustentó en un enfoque comprensivo interpretativo, a partir de una perspectiva cualitativa, consistente con el interés de acceder a una comprensión más compleja y profunda del fenómeno de estudio (Congreso Panamericano de Educación Física realizado en México en 1982), que nos coloque ante la posibilidad de contribuir a sumar conocimiento 
interpretativo. Siguiendo la escritura de otros trabajos (Scharagrodsky, 2021a, 2021b, 2021c), el enfoque analítico se fundamentó a partir de una perspectiva socio-histórica con énfasis en la historia social de los saberes y expertos (Neiburg y Plotkin, 2004; Heilbron, Guilhot, Jeanpierre, 2008; Altamirano, 2013) en diálogo con la historia de la educación física como disciplina escolar (Goodson, 2003; Scharagrodsky, 2011).

En función al universo indagado (el congreso panamericano realizado en México en 1982), se han analizado tres dimensiones constitutivas de la disciplina educativa producidas en dicho espacio académico: los saberes y perspectivas transmitidas y puestas en circulación, los expertos disciplinares más reconocidos y las instituciones legitimadoras.

Para llevar a cabo el trabajo se indagaron desde el análisis crítico del discurso (Fairclough, 2003) una serie de fuentes primarias entre las que destacaron las memorias e informes del congreso, las exposiciones centrales de los expertos, las ponencias generales, la prensa y las memorias vinculadas a otros congresos panamericanos. Asimismo, se han realizado un conjunto de entrevistas semiestructuradas (Vasilachis de Gialdino, 2007).

El análisis crítico del discurso ha articulado el "texto" junto con las condiciones históricas de producción e interacción del universo indagado (Fairclough, 2003). Las entrevistas permitieron construir categorías teóricas vinculadas con las dimensiones analizadas a partir de recurrencias y disonancias de sentido en función a grados de saturación (Vasilachis de Gialdino, 2007). El análisis permitió triangular y potenciar analíticamente las interpretaciones obtenidas de las fuentes documentales e identificar más claramente las dimensiones indagadas: los saberes y perspectivas abordadas, los referentes o expertos de la especialidad y las instituciones nacionales e internacionales actuantes. Los criterios de selección para los informantes entrevistados estuvieron vinculados con el tipo de participación y función ejercida en el congreso: organizadores y asistentes. ${ }^{3}$

Entre los interrogantes que se han planteado, son posibles de mencionar las siguientes preguntas: ¿cuáles fueron los saberes y perspectivas que sustentaron los tópicos y debates tratados en el evento internacional? y ¿quiénes fueron los expertos y las instituciones internacionales que se arrogaron la autoridad y la legitimidad del saber en la comunidad de profesores de educación física durante el congreso?

\footnotetext{
${ }^{2}$ Se han hecho entrevistas y conversaciones -virtuales- con cuatro personas. Un entrevistado estuvo vinculado con la organización del congreso (Abraham Ferreiro Toledano) y tres entrevistados ejercieron la función de asistentes durante el congreso (Héctor Icaza, José Luis Ibarra y Rodrigo Santoyo).
} 


\section{Resultados}

\section{El Congreso: Circulación de Significados y Enfoques Teóricos}

Como en anteriores ocasiones, el VIII Congreso Panamericano de Educación Física organizado en la capital mexicana tuvo el apoyo político y económico de distintas organizaciones y estructuras gubernamentales. Entre ellas se destacaron el Consejo Mexicano de Salud, Educación Física y Recreación, la Subsecretaría del Deporte, la Secretaría de Educación Pública, el Comité Olímpico Mexicano y la Confederación Deportiva Mexicana. Asimismo, la prensa tuvo un papel central en la difusión del evento. Como mencionó uno de los principales organizadores "teníamos muchos amigos en la prensa que nos ayudaron en la difusión del congreso" (Abraham Ferreiro Toledano, comunicación personal, 24 de septiembre, 2021). La cobertura por parte de periódicos como El Sol de México, El Nacional, Excélsior, El Universal, La Afición, Estadio, La Prensa, Rotativo, Esto, El Día, entre otros, fue muy profusa antes y durante el evento internacional identificando más de 30 notas vinculadas al tema.

Entre las máximas autoridades presentes en la inauguración del multitudinario evento estuvo el presidente mexicano, José López Portillo. La figura presidencial, al igual que el resto de las autoridades presentes en la inauguración, utilizaron el significante 'educación integral' con el fin de prestigiar, posicionar y legitimar la importancia de la educación física y los deportes en las sociedades modernas. La autoridad presidencial mexicana mencionó: "lo importante es la educación física como expresión cultural del hombre: basta el cultivo de la misma, de la armonía mental, para que satisfaga el propósito" (Memorias del VIII Congreso Panamericano de Educación Física, 1982, p. 11). Por otra parte, el Subsecretario del Deporte, Dr. Manuel Mondragón y Kalb señaló:

En México, como en el resto de los países (americanos) estamos convencidos de que la educación del hombre es integral; y en este concepto debemos considerar a la educación física, porque gracias a ella, aseguramos para los niños jóvenes de América, la salud y la fortaleza que requieren nuestros pueblos (...). (Memorias del VIII Congreso Panamericano de Educación Física, 1982, p. 11)

Esta misma retórica que articuló integralidad y salud, esgrimió el Dr. Carlos Vera Guardia, presidente del Comité Permanente de Planificación de los panamericanos y figura preponderante en la organización de estos eventos: “(...) los que trabajamos por la educación física tenemos la enorme y sagrada responsabilidad de trabajar para la formación integral del ser humano, el capital más importante y el único fundamental de nuestros jóvenes pueblos de América" (Memorias del VIII Congreso Panamericano de Educación Física, 1982, p. 13). (Figura 1) 


\section{Figura 1}

Inauguración del VIII Congreso Panamericano de Educación Física

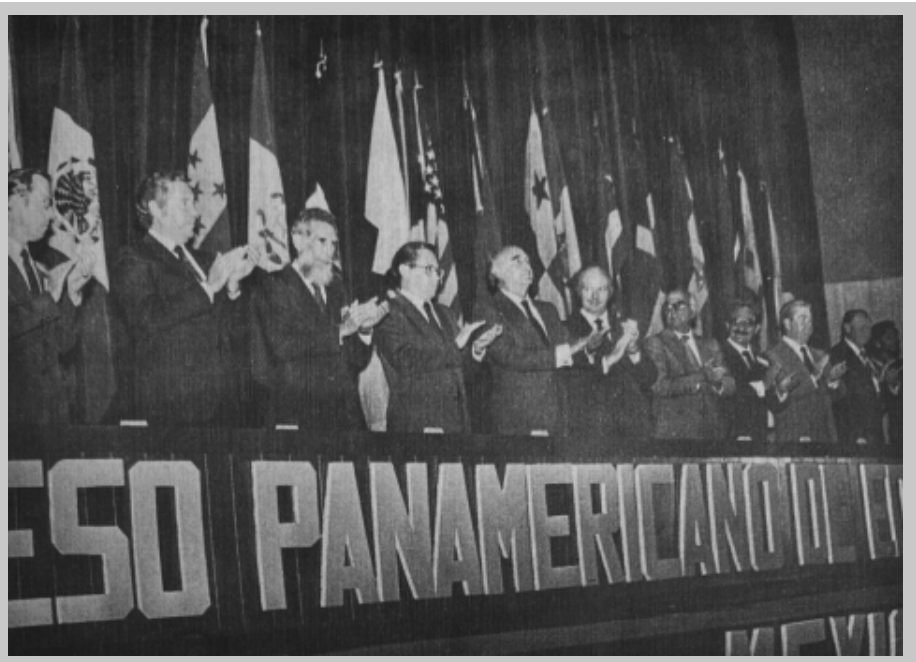

"Lo importante es la Educación Física como expresión cultural del hombre, basta el cultivo de la misma, de la armonía mental para que satisfaga el propósito"

\section{LIC. JOSE LOPEZ PORTILLO}

Nota: Palabras del presidente mexicano y demás autoridades. Consejo Mexicano de Salud, Educación Física y Recreación (1982). Memoria del VIII Congreso Panamericano de Educación Física. Publicado por la Subsecretaría del Deporte, p. 3.

Con el apoyo de dichas instituciones, autoridades y a partir de una retórica poco original y claramente decimonónica (Scharagrodsky, 2011), se realizó en la Escuela Superior de Educación Física (ESEF) y en el Instituto Mexicano del Seguro Social, en el distrito federal, el congreso panamericano. Como mencionaron varios entrevistados el apoyo estatal fue muy importante para concretar el congreso: "Fue un evento multitudinario apoyado por el Estado mexicano. De hecho estuvo el mismísimo presidente y otras autoridades deportivas y educativas" (José Luis Ibarra Mercado, comunicación personal, 23 de septiembre, 2021).

El evento internacional se realizó en un contexto educativo mexicano caracterizado por una fuerte expansión del mismo en los años ‘70, que incluyó la difusión de actividades físico-deportivas. En esa década se amplió

el Sistema Educativo Nacional, primero en un sentido eminentemente cuantitativo, que se compendiaba en la fórmula más maestros, más escuelas, en todos los niveles educativo; pero también por la incorporación de grupos, sectores y comunidades marginadas, y nuevas modalidades, como la educación bivalente y los programas de profesionalización temprana. Entre 1970 y 1976, la matrícula en todos los niveles aumentó 
un 44\%, poco más de 5 millones de estudiantes. Esto fue posible por tres acciones: un incremento en la oferta educativa, lo que se denominó en la época más escuelas y más maestros, principalmente en las escuelas federales, la vía extensiva del crecimiento de la oferta; también nuevas escuelas que articulan la formación pedagógica con la técnica, la vía intensiva de formación profesional; y nuevos programas en educación media superior (bachillerato), superior y de posgrado, en la terminología del momento, la vía modernizadora. (González Villarreal, 2018, p. 102)

Por otra parte, la Secretaría de Educación Pública -responsable de la educación en México-, se desconcentró en el año 1978.

La desconcentración fue planteada en términos de reorganización en el nivel administrativo, como factor de desarrollo y equilibrio para la nación. En este sentido, planteaba reducir la hegemonía del centralismo en el desarrollo y operación del sistema educativo. Con esto se establecieron Delegaciones Generales Estatales que tendrían como objetivo operar y coordinar los servicios educativos en las regiones estatales. La década de los ochentas marcó un antes y un después en el sistema educativo gracias a las reformas efectuadas en la materia como la reestructuración de orden normativo, político, social y educativo, a través de las reformas de Primera, Segunda y Tercera generación. Las reformas de primera generación hacen alusión a la descentralización y al financiamiento de la educación, la segunda generación habla de la evaluación de la calidad del sistema educativo, mientras que las de tercera generación contienen las transformaciones del espacio escolar. (Gómez Collado, 2017, p. 145)

En el marco de estos procesos educativos en 1982 la educación física y los deportes adquirieron una nueva visibilidad (Ferreiro Toledano, 2006). El congreso se convirtió en un espacio de sociabilidad e intercambio masivo y heterogéneo en términos de nacionalidades presentes: mil cien asistentes, provenientes de veinticuatro países (Xicotencatl, 21 de julio, 1982): Antillas Holandesas, Argentina, Bolivia, Brasil, Canadá, Colombia, Costa Rica, Chile, Ecuador, España, Estados Unidos, Guatemala, Honduras, Jamaica, Nicaragua, Panamá, Paraguay, Perú, Puerto Rico, República Dominicana, El Salvado, Surinam, Venezuela y México (Memorias del VIII Congreso Panamericano de Educación Física, 1982, pp. 36-42). Como mencionó un entrevistado "el evento se diferenció de otros panamericanos (...) fue un éxito por la cantidad de asistentes, delegados de países, personalidades mundiales de la educación física y el deporte y el presidente de México (...) fuimos noticia en muchos periódicos mexicanos" (Rodrigo Santoyo, comunicación personal, 7 de julio, 2021). 
Siguiendo la tradición de otros congresos, en México se realizaron varias actividades deportivas, culturales y folklóricas entre las que sobresalieron "los bailes tradicionales mexicanos (...) si mal no recuerdo bailes de Jalisco y Veracruz (...) y distintas actividades deportivas realizadas en el gimnasio central del ESEF” (Héctor Icaza, comunicación personal, 22 de septiembre, 2021). Otro asistente mencionó "el brillo de los bailes realizados en la inauguración a cargo del grupo del profesor Federico Vidales (...) un grupo de danza folklórica llamado iyolmazehuayotl (la danza en el alma) (...) un grupo muy conocido" (José Luis Ibarra Mercado, comunicación personal, 23 de septiembre, 2021). A pesar de que parte de la retórica del evento estuvo vinculada con la hermandad americana y la salud de los pueblos americanos, al mismo tiempo, a partir de este tipo de actividades se reafirmaron las identidades y particularidades locales y regionales.

Analizar los diferentes temas tratados y las formas de abordarlos nos brindan indicios sobre cuáles fueron las discusiones y problemáticas que los expertos, referentes y parte de la comunidad de profesores de educación física, médicos, maestros y dirigentes deportivos, realizaron durante dicho congreso.

Los ejes temáticos del congreso fueron seis: 1."el Perfil del educador físico”, 2."la formación de docentes para la educación física, recreación y deportes”, 3.“los planes y programas: preescolar, primaria y educación media", 4."la investigación en la educación física”, 5."la Educación física y deportes: problemas permanentes” y 6. "temas libres” (Memorias del VIII Congreso Panamericano de Educación Física, 1982, p. 20).

El primer eje con conferencistas centrales provenientes de México (profesor Teocrático Arteaga Nochebuena de la Escuela Superior de Educación Física -ESEF-), Estados Unidos (Dr. Peter Everett, presidente de la International Council for Health, Physical Education, Recreation, Sport and Dance -ICHPER-) (Figura 2) y Venezuela (Dr. Boris Planchart Oraá del Instituto Universitario pedagógico de Caracas), luego de las discusiones y debates recomendaron definir el perfil docente ideal, a partir de dos tipos de características: las "profesionales (eficiencia en la administración, capacidad en la investigación, competencia en la conducción del proceso educativo y eficiencia en la promoción de la salud) y personales (vocación, liderazgo, condiciones óptimas de salud, presentar una aptitud física general, adecuada escala de valores y espíritu de superación personal) (Memorias del VIII Congreso Panamericano de Educación Física, 1982, pp. 20, 27). Como en décadas pasadas, el perfil del profesor de educación física deseable no solo debía incorporar determinadas competencias y saberes sobre su actividad, sino demostrar cierta moral 'decente' y una 'adecuada' apariencia física. Ser y parecer fueron, a lo largo de la historia, una constante en la definición dominante sobre el modelo de un buen profesor de educación física. 


\section{Figura 2}

El Dr. Peter Everett y demás autoridades durante el congreso

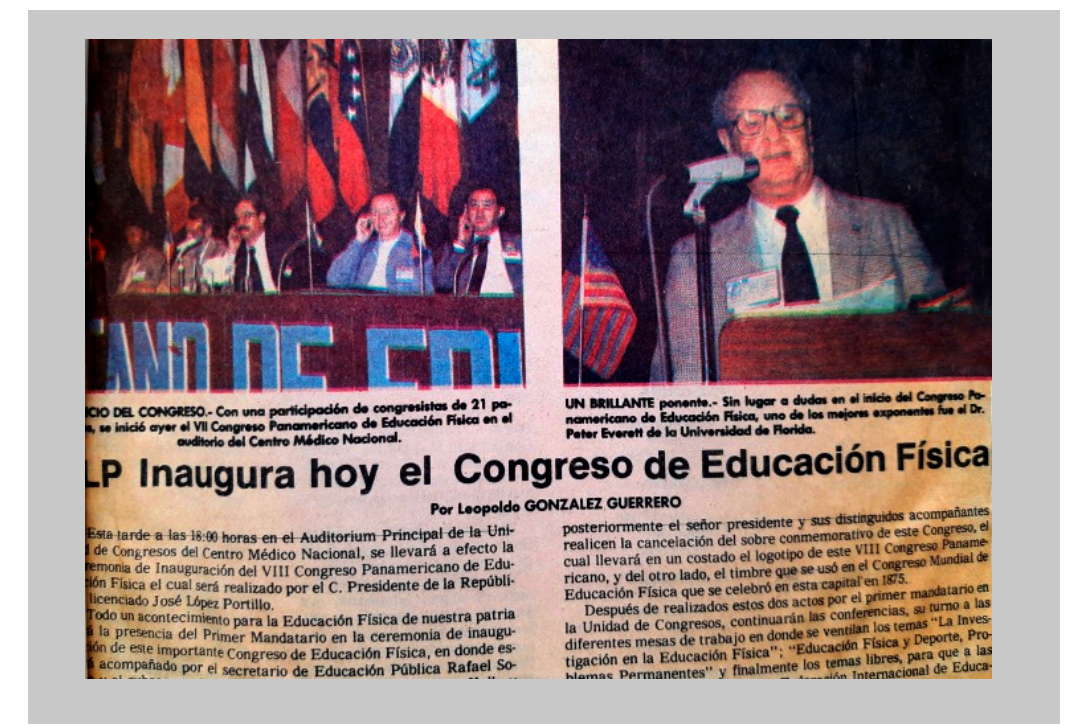

Nota: González Guerrero, L. (1982, 20 de julio). Presidente de México, JLP, Inaugura hoy el Congreso de Educación Física. El Sol de México, México.

El segundo eje con conferencistas centrales provenientes de México (profesor José Antonio Solís de la ESEF), Chile (profesor Carlos Gómez Aros, presidente de la Corporación Nacional Universitaria de Deportes de Chile) y Canadá (Dr. Fred Martens de la Universidad de Victoria) discutió la “(...) falta de personal calificado en gran parte del continente americano” y propuso “incrementar el número de escuelas formadoras de docentes en educación física”, así como "darle importancia al diseño curricular de programas para la formación de docentes, y a la investigación (...)" (Memorias del VIII Congreso Panamericano de Educación Física, 1982, pp. 20, 27-28). Este eje consensuó la necesidad de incrementar la presión sobre las diversas reparticiones del Estado y otros actores privados, con el fin de aumentar en cantidad y calidad los centros de formación docente de la especialidad. La capacidad de 'lobby' de este grupo ocupacional se incrementó en los '80 y '90 en muchos países latinoamericanos, obteniendo el control no solo de los centros de formación docente, sino de estructuras gubernamentales vinculadas con las políticas deportivas regionales y nacionales. De acuerdo a uno de los máximos responsables en la organización del congreso, para el caso mexicano, el evento internacional "Generó cambios para la educación física ya que hubo más apoyo a las escuelas superiores de educación física y recursos para el cumplimiento de la obligatoriedad de la educación física" (Abraham Ferreiro Toledano, comunicación personal, 24 de septiembre, 2021) 
El tercer eje con conferencistas centrales provenientes de México (profesor Marco Antonio Escalante, Director General de Educación Física), Canadá (Dr. Davidson Stewart, vicepresidente de la ICHPER) y Costa Rica (Dra. Martha Picado de la Universidad de Costa Rica) debatió los mejores modos sobre cómo elaborar y planificar un diseño curricular, el cual debía incluir "las teorías de la enseñanza y aprendizaje", "las estrategias y procedimientos de instrucción" y "la evaluación”. Entre las recomendaciones se mencionaron que los planes “(...) no se desvíen hacia el deporte competitivo (...)", tener en cuenta "los diferentes niveles educativos" e "incorporar dentro de la estructura del plan y programa, un proceso de evaluación que permita objetivizar sus resultados" (Memorias del VIII Congreso Panamericano de Educación Física, 1982, pp. 20, 28-29). Varias de estas preocupaciones estuvieron presentes en los primeros panamericanos desarrollados en los años '40, en donde se denunció y cuestionó los peligros del "campeonismo deportivo" (Scharagrodsky, 2021a). La objetivización y mensurabilidad de "logros a corto y mediano plazo" de los resultados en la curricula de la educación física (Memorias del VIII Congreso Panamericano de Educación Física, 1982, p. 28), fue una constante en los panamericanos de los años '60 y '70 a partir de las reflexiones dominantes brindadas por las teorías tradicionales o tecnocráticas del curriculum (Scharagrodsky, 2021b). La apelación a la educación integral como re-aseguro de la disciplina también fue un tema recurrente y apareció con fuerza en las conclusiones de varios ejes: "los planes y programas de educación física deben incorporarse en forma oficial como parte de la educación integral en la planeación educativa de cada uno de los países americanos" (Memorias del VIII Congreso Panamericano de Educación Física, 1982, p. 28).

El cuarto eje con conferencistas centrales provenientes de Brasil (Dres. Renato Brito Cunha y Herbert de Almeida de la Universidad de Río de Janeiro), de Estados Unidos (Dr. Jan Broekhoff presidente de la comisión de investigación de la ICHPER) y México (Dr. César Bravo Barajas del ESEF) discutieron a partir de un diagnóstico consensuado: la "falta de investigación” en la mayoría de los centros de formación; la necesidad de "que la investigación en educación física sea una prioridad en el ámbito educativo", buscando "apoyos económicos, políticos y privados" para su desarrollo (Memorias del VIII Congreso Panamericano de Educación Física, 1982, pp. 20, 29-30). Salvo algunos países como Estados Unidos y Canadá donde la investigación -de tipo cuantitativa y experimental- era más común y usual, en el resto de los países latinoamericanos la carencia y la falta de solidez en la investigación vinculada a la disciplina y al deporte escolar fue identificada como un déficit a resolver. 
El quinto eje con conferencistas centrales provenientes de Estados Unidos (Dra. Fay Biles de la Universidad de Kent), Colombia (Dra. Marta Moncada y Dra. Consuelo Zea del Instituto Colombiano de la Juventud y el Deporte) y México (profesor Carlos López Rubio del Consejo Nacional de Salud, Educación Física y Recreación) reflexionaron sobre la necesidad de "establecer límites precisos en el campo de acción de la educación física y el deporte (...)”. La discusión central estuvo vinculada con "la deformación del deporte en la competencia extrema y en el campeonismo". Las preocupaciones centrales fueron establecer límites claros entre ambas prácticas, especialmente en el ámbito educativo. De esta manera, se recomendó “dar prioridad en el mejor desarrollo de la educación física en nuestros países a los programas con énfasis prioritarios en las actividades de tipo educativo sobre las de tipo competitivo" (Memorias del VIII Congreso Panamericano de Educación Física, 1982, pp. 20, 30). (Figura 3)

\section{Figura 3}

\section{Distintos momentos del VIII Congreso Panamericano de Educación Física}

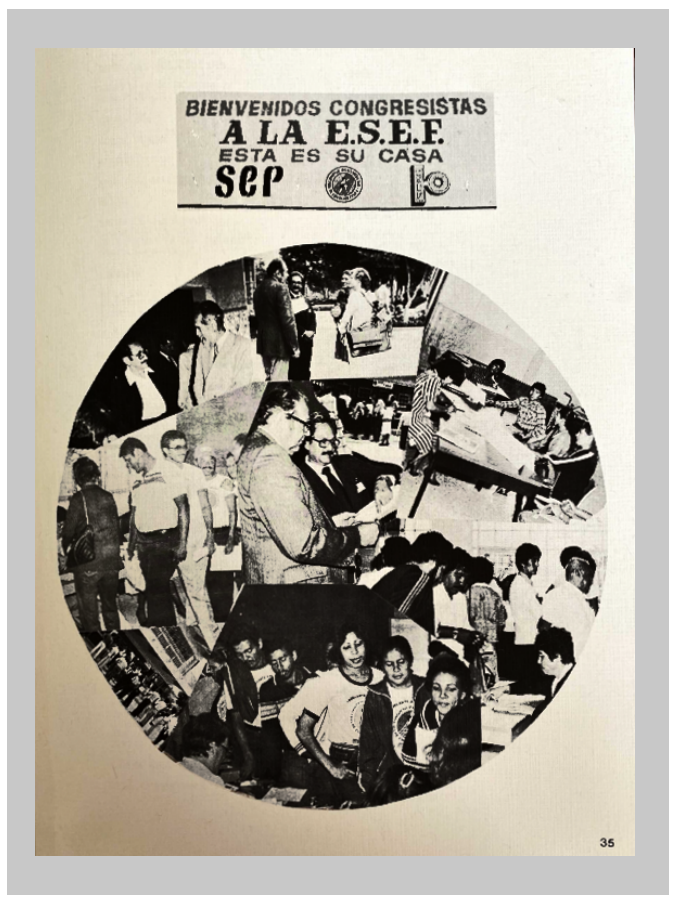

Nota: Diversas actividades durante el Congreso. Consejo Mexicano de Salud, Educación Física y Recreación (1982). Memoria del VIII Congreso Panamericano de Educación Física. Publicado por la Subsecretaría del Deporte, p. 35.

Por último, el eje seis estuvo atravesado por una variedad de temas y preocupaciones. Desde el análisis cinemático del movimiento, pasando por la necesidad de establecer criterios adecuados acerca de los exámenes físicos de salud o los problemas vinculados con la participación 
de la mujer. Con respecto a este último tema se propuso, retomando varias ponencias de mujeres norteamericanas: "tomar en consideración solo la capacidad en el desempeño profesional para la designación de altos puestos administrativos y docentes sin detenerse a pensar si es hombre o mujer". Asimismo, se reivindicó "el derecho y el deber de la mujer de participar a la par del hombre” en todo aquello vinculado con la educación física y el universo deportivo y "diseñar programas para favorecer un cambio de mentalidad a nivel de padres de familia, educadores y grupos sociales en general". Estas preocupaciones influenciadas por los movimientos feministas de los años '60 y '70, especialmente en los países anglosajones, fue disruptiva en el plano discursivo, aunque no parece haber generado grandes cambios en la educación física de muchos de los países latinoamericanos en los años ' 80 atravesados por tradiciones más refractarias a este tipo de reclamos vinculados con la igualdad de género. Aunque en México hubo importantes eventos deportivos en los años '70 como el Mundial de fútbol femenino, los estereotipos y las presiones sociales seguían estando presentes a la hora de acceder o practicar ciertos deportes por parte de niñas y mujeres (Brewster \& Brewster, 2018, p. 12).

De hecho, recién en los panamericanos realizados en Cuba en 1987 se incorporó por primera vez una mujer en el comité permanente de planificación (órgano central de decisión) y recién en los años '90 hubo mayor visibilidad desde una perspectiva crítica de estos temas.

\section{El Congreso: sobre el Derecho a Hablar y acerca del Encuadre Institucional}

El congreso realizado en México no solo instaló, fabricó y naturalizó ciertos temas como constitutivos de la disciplina, sino que dicha operación necesitó de determinadas figuras para consagrar la 'verdad' -pedagógica, didáctica, biomédica o psico-educativa- de la disciplina: los expertos. Estas figuras -materiales, imaginarias y simbólicas- funcionaron en el congreso como los grandes directores de orquesta a la hora de expresar ciertas melodías cargadas de sentidos y significados adecuados, racionales y científicos. De alguna manera, los expertos sobre la educación -y la salud- de los cuerpos en movimiento describieron y explicaron algo que este selecto grupo a partir de ciertos discursos creó. Primero crearon y después descubrieron, pero, por un artificio retórico, aquello que crearon terminó apareciendo como un descubrimiento (Butler, 2002). En este sentido, lo que hicieron los expertos, fue crear una noción particular de la educación física, los deportes y la recreación. Aquello que los expertos afirmaron que era la salud, la infancia, la motricidad, el profesor de educación, la formación docente o la curricula deportiva pasó, de hecho, a ser la salud, la infancia, la motricidad, el profesor de educación, la formación docente o la curricula deportiva. (Figura 4) 


\section{Figura 4}

Logo del Congreso con una figura de un cuerpo infantil ideal

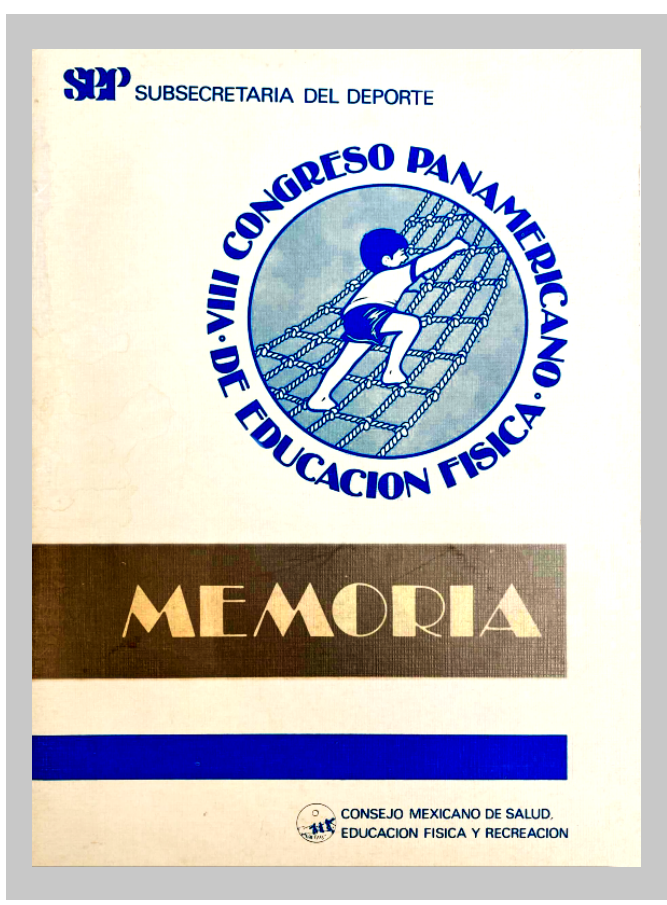

Nota: Imagen de una infancia sana. Consejo Mexicano de Salud, Educación Física y Recreación (1982). Memoria del VIII Congreso Panamericano de Educación Física. Publicado por la Subsecretaría del Deporte, Tapa.

Los expertos se erigieron en estos espacios no solo como las voces autorizadas y legitimadas por la propia comunidad, sino también por otros actores sociales interesados (instituciones deportivas, organizaciones sanitarias, estructuras educativas, editoriales, prensa especializada, prensa generalista, etc.). El congreso internacional consolidó algunas voces y, al mismo tiempo, deslegitimó otras configurando un campo enunciativo en donde ciertas posiciones materializadas en determinados actores sociales, ejercieron mayores cuotas de poder, influencia y autoridad. Quienes 'tomaron' la palabra no solo transmitieron ciertas categorías para interpretar e interpelar a la realidad disciplinar como las mejores y las más adecuadas, sino que al mismo tiempo excluyeron deliberadamente otras grillas interpretativas. Como en cualquier espacio en el que circularon y se transmitieron saberes, hubo expertos con funciones y competencias diferenciadas. Por un lado, estuvieron los "expertos académicos". Por el otro, los “expertos en gestión” y los “expertos en formación”. 4

\footnotetext{
${ }_{3}^{3}$ La presente clasifícación adopta como criterio las actividades y funciones más visibles de los expertos en la especialidad. No obstante, la clasificación es porosa y sus fronteras son permeables ya que la multifunción era común en muchos de ellos. En varios casos, un "experto académico" realizaba trabajos de formación para alguna institución universitaria, o algún "experto en formación" se dedicaba también a la gestión. Aunque había varias combinaciones posibles, los expertos sobresalían y eran socialmente reconocidos por el resto de la comunidad en algunas tareas o funciones en particular.
} 
Entre los expertos mexicanos se destacaron en la organización los doctores Manuel Mondragón Kalb, Marco Antonio Taracena, Fabio Gerard Zubia, Jorge Ojanguren Preciado, José García Ocampo y los profesores Abraham Ferreiro Toledano, Teresa Morales Mosqueda, Víctor Manuel Lonngy, Jesús Aguilar, Patricia Hoffman, Norberto Pacheco Arreguin, Julián Medrano Pineda, César Moreno Bravo, Santiago Medina Candanedo, Jorge Almeida Leva, Eduardo Gorraez Larrinaga, Carlos López Rubio, Noemí Mandujano, Lidia Vaca Aldapa, Alfonso Taboada Argaez, Jaime Velázquez Resendiz, Alfonso Espinoza y María Eugenia Contreras. El primero fue presidente del comité organizador y el resto tuvo diferentes funciones dentro del congreso (vicepresidencia, secretaria general, coordinación de ponencias, etc.). Algunos se venían destacando en el terreno internacional como Abraham Ferreiro Toledano -clave en el congreso y referente histórico mexicano- quien desde finales de los años '50 se venía desempeñando como el Primer Delegado General de la Federation International d'Education Physique (FIEP) en México. Otros como Julián Medrano Pineda sobresalió en un deporte como el atletismo, siendo responsable de dicha actividad en los Juegos Olímpicos de 1968 y en los Panamericanos de 1975. Otros como César Moreno Bravo estuvo vinculado con la formación docente de la especialidad habiéndose desempeñado como director general de la Escuela Nacional de Educación Física entre 1964 y 1980 y, por último, otros estuvieron vinculados con las estructuras estatales deportivas como Eduardo Gorraez Larrinaga que trabajo en la Comisión Nacional de Cultura Física y Deporte mexicana (CONADE).

Entre los expertos extranjeros estuvieron presentes referentes disciplinares de cada uno de sus países como el Dr. Carlos Vera Guardia y el Dr. Boris Planchart Oraa por Venezuela, la Dra. Sonia Toro Seda por Puerto Rico, la profesora Josefa Lora Risco por Perú, el Dr. Peter Everett, el Dr. Jan Broekhoff, el Dr. Carl A. Troester y la Dra. Fay Biles por los Estados Unidos, el Dr. Fred L. Martens y el Dr. Stewart A. Davidson por Canadá, la Dra. Martha Picado por Costa Rica, el Dr. Renato Brito Cunha, el Dr. Francisco Camargo Netto, el profesor Jacinto Targa y el Dr. Herbert de Almeida por Brasil, la Dra. Consuelo Zea y Martha Moncada por Colombia, el profesor Carlos Gómez Aros por Chile, entre otros. Entre las figuras europeas sobresalieron el francés Dr. Pierre Seurin y el belga Dr. Marcel Hebbelinck.

Entre los “expertos académicos" se destacaron, entre otros, las exposiciones del norteamericano Dr. Anthony Annarino o la peruana Josefa Lora Risco. El primero tuvo una prolífica producción y una gran trayectoria académica en su país (Templin \& Harper, 2011). Se doctoró en los años ‘60 en la Universidad de Indiana y trabajó en la prestigiosa Universidad de Purdue. Entre sus libros se destacaron Teaching tumbling in physical education de 1960, Developmental conditioning for 
physical education and athletics de 1972 y Curriculum theory and design in physical education de 1980. La segunda se destacó en el campo de la educación psicomotriz extendiendo su influencia más allá de las fronteras peruanas (Limache, 2018).

De igual manera hubo, como en los anteriores panamericanos, "expertos en gestión” y "expertos en formación”. Los “expertos en gestión” se encargaron de administrar e implementar desde importantes instituciones estatales u organizaciones sin fines de lucro -nacionales o internacionales- políticas deportivas y recreativas de alto impacto social. Entre los más destacados expertos en gestión estuvieron presentes difundiendo las bondades y beneficiosos de ciertas políticas deportivas internacionales el francés Dr. Pierre Seurin quien se venía desempeñando como presidente la Federation International d'Education Physique (FIEP) desde 1970, el belga Dr. Marcel Hebbelinck presidente de la poderosa e influyente International Council for Health, Physical Education, Recreation, Sport and Dance (ICHPER), o los norteamericanos Carl A. Troester -integrante clave de la American Alliance for Health, Physical Education, Recreation and Dance's (AAHPER)- y la Dra. Carole Oglesby que, con otro perfil político, desde la década de 1960 se convirtió en una fuerte defensora del deporte femenino y de las disidencias sexuales (fue unas de las primeras mujeres en el atletismo norteamericano en declarase lesbiana y 'defender' dicha causa de manera pública). En los años ‘70, participó activamente en una serie de proyectos de formación y empoderamiento de entrenadoras en América Central y del Sur. Varios de los entrevistados recordaron en especial a "Pierre Seurin y la importante charla que ofreció" (Héctor Icaza, comunicación personal, 22 de septiembre, 2021). El director del VIII Congreso Panamericano, Abraham Ferreiro Toledano, no sólo mencionó la importante presencia de Seurin, sino de varias instituciones internacionales "Además de figuras como Seurin, quien dirigió una importante actividad deportiva en el ESEF (...) dictó una clase de gimnasia, y enseñó principios del atletismo (...) hubo muchas instituciones internacionales que estuvieron en el congreso" (Abraham Ferreiro Toledano, comunicación personal, 24 de septiembre, 2021).

En cualquier caso, la importancia de estos "expertos en gestión" y de las instituciones internacionales mereció una resolución general por parte de los congresistas, la cual fue difundida en varios medios de prensa (Zaragoza, 23 de julio, 1982, p. 17):

Recomendar a la UNESCO, OEA y demás Organizaciones Internacionales que promuevan programas de educación física, recreación y deportes en todos los países de su área de influencia, considerando que estas actividades son un derecho fundamental de todos los seres humanos y que establezcan contactos permanentes con el Congreso Panamericano de Educación Física, ICHPER, FIEP, AIESEP, IAPESGW y otras instituciones de 
carácter internacional, con el objeto de llegar a todos los profesores de educación física y profesionales afines. (Memorias del VIII Congreso Panamericano de Educación Física, 1982, p. 33)

Por último, los “expertos en formación” se dedicaron muy especialmente a la docencia de los futuros profesores y profesoras de educación física, de los entrenadores, de los dirigentes y de los especialistas en el quehacer deportivo. Todo ello en un contexto internacional de creciente profesionalización de la formación superior de la educación física (Silva \& Molina Bedoya, 2015). Algunos de los expertos en formación más reconocidos en el congreso fueron el canadiense Dr. Fred L. Martens y la costarricense Martha Picado. El primero enseñó Educación Física en Victoria College y jugó un papel decisivo en el establecimiento de la Escuela de Educación Física en la Universidad de Victoria, Canadá. La segunda fue una de las figuras centrales en la formación de profesores de educación física en la Escuela de Educación Física de la Universidad de Costa Rica. Su sólida formación realizada en los años '70 en la República Federal Alemana y en Estados Unidos (Temple University y Springfield College) la convirtió en una experta de referencia en la formación no solo en Costa Rica (fue la primera persona en obtener un doctorado en su país), sino en varios países de centro-américa (Araya Vargas, \& Claramunt Garro, 2020, p. 23). Ellos, entre muchos otros, sobresalieron en la formación docente durante varias décadas.

Además de las instituciones y organismos internacionales mencionados, hubo presencia de varios docentes e investigadores provenientes de espacios universitarios como los de Venezuela, Costa Rica, Colombia, Brasil, Canadá y, muy especialmente de los Estados Unidos: el Instituto Universitario Pedagógico de Caracas en Venezuela, la Universidad Pedagógica Nacional en Colombia, la Universidad de Ottawa y de Victoria en Canadá, la Universidad de Costa Rica, la Universidad de Río de Janeiro y la Universidad Federal de Rio Grande do Sul en Brasil, la Universidad de Oregon, de Kent, de Austin, de Purdue, de Florida, etc. en los Estados Unidos, entre otras. Este tipo de presencia se fue consolidando en los panamericanos organizados a finales de los ' 80 y en la década de los '90 del siglo XX, mostrando la importancia que tuvieron estas instituciones (Universidades, ONGs, organismos internacionales, etc.) a la hora de potenciar el amplio universo de la cultura física y proyectar políticas deportivas y recreativas a grandes porciones de la población. 


\section{Conclusiones}

El VIII Congreso de Educación Física fue un éxito en términos de asistencia, apoyo estatal y países presentes. Las diferentes áreas de trabajo problematizaron distintas dimensiones de la disciplina y del fenómeno deportivo en las instituciones educativas. Algunos temas fueron, con matices, abordados en los panamericanos anteriores. Por ejemplo, la preocupación por formar un perfil docente donde el peso moral fuese un aspecto central para el ejercicio de la condición docente, el rechazo al "campeonismo deportivo" o la búsqueda de criterios uniformes para la medición física fueron una constante. También, abundaron, como en otros panamericanos, la identificación de ciertas carencias de la disciplina: las dificultades en la formación de profesores de educación física competentes o la ausencia de investigación, entre otras cuestiones. La poco novedosa retórica de la integralidad (educación integral) fue una de las metáforas más utilizadas con el fin de legitimar a la disciplina dentro y fuera de la institución educativa. La tradición tecnocrática vinculada con la elaboración curricular también fue un tema indagado en congresos anteriores. Pero, al mismo tiempo, este congreso introdujo tópicos y enfoques nuevos. En especial la cuestión del deporte femenino y la igualdad de género marcaron un quiebre a partir de este congreso. Si bien los cambios fueron lentos, la cuestión femenina y la equidad comenzaron a instalarse con mayor fuerza en estos congresos. Otros temas se amplificaron como, por ejemplo, el tratamiento y la visibilización del importante papel que tenían las instituciones internacionales dedicadas a la promoción de la educación física, los deportes y la recreación. También crecieron las inquietudes vinculadas con la necesidad de implementar políticas deportivas masivas con apoyos no solo del Estado, sino también de actores de la sociedad civil.

Estas diversas preocupaciones fueron elaboradas por expertos "académicos", en "gestión" y en "formación", estrechamente vinculados con diversas instituciones nacionales e internacionales relacionadas con el campo de la educación física y los deportes. Los expertos y las instituciones internacionales no solo prestigiaron al evento, sino que regularon y controlaron los sentidos ‘verdaderos' vinculados el universo deportivo, gímnico y recreativo moderno. En síntesis, el congreso internacional realizado en México en 1982 fue parte de un circuito más general con una larga historia, que lentamente naturalizó ciertas necesidades (más salud, más movimiento, más práctica deportiva, más recreación, etc.) y consolidó a un grupo ocupacional compuesto no solo por profesores de educación física, sino también por médicos deportólogos, entrenadores, dirigentes deportivos, etc. Todos ellos, con el tiempo se volvieron importantes a la hora de gestionar la salud y la educación de los cuerpos individuales y de las poblaciones en las sociedades modernas. 


\section{Referencias Bibliográficas}

Altamirano, C. (2013). Intelectuales. Notas de investigación sobre una tribu inquieta. Siglo XXI.

Araya Vargas G., \& Claramunt Garro, M. (2020). Actividad física en Costa Rica, antecedentes históricos y revisión de sus evidencias cientificas en el país. Ministerio de Salud y Universidad de Costa Rica, Escuela de Educación Física y Deportes.

Brewster, C., \& Brewster, K. (2018). Women, Sport, and the Press in Twentieth-Century Mexico. The International Journal of the History of Sport, 35(10), 965-984. doi.org/10.1080/095233 67.2019.1575816

Butler, J. (2002). Cuerpos que importan. Sobre los límites materiales y discursivos del “sexo”. Paidós.

Comisión Nacional de Educación Física (1950). Informe del III Congreso Panamericano de Educación Física. Editada por la Comisión Nacional de Educación Física (CNEF).

Consejo Mexicano de Salud, Educación Física y Recreación (1982). Memoria del VIII Congreso Panamericano de Educación Física. Publicado por la Subsecretaría del Deporte.

Consejo Mexicano de Salud, Educación Física y Recreación (1982). Memoria del VIII Congreso Panamericano de Educación Física. Subsecretaría del Deporte.

Dirección de Deporte (1976). Acta final del VI Congreso Panamericano de Educación Física y II Gimnasiada Americana, Tomo I. Publica Universidad de Carabobo.

Fairclough, N. (2003). El análisis crítico del discurso como método para la investigación en ciencias sociales. En R. Wodak, y M. Meyer, (Comp.), Métodos de análisis crítico del discurso (pp. 179-204). Gedisa.

Ferreiro Toledano, A. (2006). Desarrollo de la Educación Física y el Deporte en México en el siglo XX. (1889-2000). Tomo II. Comité Olímpico Mexicano.

Ferriz, D. (1965). Documento de Trabajo No 24. Memorias del IV Congreso Panamericano de Educación Física, s/e.

Gómez Collado, M. E. (2017). Panorama del sistema educativo mexicano desde la perspectiva de las políticas públicas. Innovación Educativa, 17(74), 143-163.

González Guerrero, L. (1982, 20 de julio). Presidente de México, JLP, Inaugura hoy el Congreso de Educación Física. El Sol de México, México. 
González Villarreal, R. (2018). La reforma educativa en México: 1970-1976. Espacio, Tiempo y Educación, 5(1), pp. 95-118. doi: http://dx.doi.org/10.14516/ete.214

Goodson, I. (2003). Estudio del currículum. Casos y métodos. Amorrortu Editores.

Heilbron, J., Guilhot, N. y Jeanpierre, L. (2008). Toward a transnational history of the social sciences. Journal of the History of the Behavioral Sciences, 44(2), 146-160. https://doi.org/10.1002/ jhbs. 20302

Kirk, D. (2010). Physical Education Futures. Routledge.

Limache, M. (2018). Heroína de la educación psicomotriz: Josefa Lora Risco. Revista con la A, 59, 1-4. Recuperado de https:/conlaa.com/heroina-de-la-educacion-psicomotriz-josefa-lora-risco/

Linhales, M., Rodrigues Puchta, D. \& Rosa, M. C. (org.) (2019). Diálogos Transnacionais na História da Educação Física. Fino Traço.

Ljunggren, J. (2011). ¿Por qué la gimnasia de Ling? El desarrollo de la gimnasia sueca durante el siglo XIX. En P. Scharagrodsky (org.), La invención del homo gymnasticus (pp. 37-51). Prometeo Editorial.

Ministerio da Educação e Saúde (1945). Anais Primero Congreso Panamericano de Educação Física, Volume I. Imprensa Nacional.

Ministerio de Cultura y Educación (1971). Memoria del V Congreso Panamericano de Educación Física. Desarrollo y relatos. Talleres Gráficos Yunke.

Neiburg, F., y Plotkin, M. (Comps.) (2004). Intelectuales y expertos. La constitución del conocimiento social en la Argentina. Paidós.

Scharagrodsky, P. (2021c). Saberes, expertos e instituciones vinculadas al universo del movimiento. El caso del VII Congreso Panamericano de Educación Física, Dominicana 1980. Ciencia y Educación, 5(3), 145-160. doi: https://doi.org/10.22206/cyed.2021.v5i3.pp145-160

Scharagrodsky, P. (2021a). Cartografiando saberes, grupos ocupacionales, instituciones, agentes y redes. El caso del Segundo Congreso Panamericano de Educación Física, México, 1946. Revista Mexicana de Historia de la Educación, 9(17), 118-142. https://doi.org/10.29351/rmhe.v9i17.336

Scharagrodsky, P. (2021b). Cuerpos, políticas y pedagogías en disputa. El Quinto Congreso Panamericano de Educación Física, Buenos Aires, 1970. Revista Contemporánea, 14(1), 146163. Recuperado de https://ojs.fhce.edu.uy/index.php/cont/article/view/1097 
Scharagrodsky, P. (2011). Presentación. En P. Scharagrodsky (org.), La invención del homo gymnasticus. (pp. 15-20.). Prometeo Editorial.

Secretaría de Estado, Educación, Bellas Artes y Cultos (1980). Memoria oficial del VII Congreso Panamericano de Educación Física. Publicado por la Secretaría de Estado.

Secretaría de la Defensa Nacional (1946). Memoria oficial del II Congreso Panamericano de Educación Física. Publicada por la Dirección Nacional de Educación Física y Enseñanza Premilitar.

Silva, A. M. \& Molina Bedoya, V. (2015). Formação Profissional em Educação Física na América Latina: Encontros, Diversidades e Desafios. Paco Editorial.

Templin, T. J., \& Harper, W. A. (2011). Anthony A. Annarino, 1923-2010. Journal of Physical Education, Recreation \& Dance, 82(1), 7-50. doi: 10.1080/07303084.2011.10598552 https:// www.tandfonline.com/doi/abs/10.1080/07303084.2011.10598552?journalCode=ujrd20

Vasilachis de Gialdino, I. (2007). Estrategias de investigación cualitativa. Gedisa.

Vigarello, G., \& Holt, R. (2005). El cuerpo cultivado: gimnastas y deportistas en el siglo XIX. En A. Corbin, J. Courtine \& G. Vigarello (dir.), Historia del cuerpo. De la Revolución francesa a la Gran guerra (pp. 295-354). Volumen II. Taurus.

Xicotencatl, A. (1982, 21 de julio). Reúne el Congreso a 1100 educadores de 23 países. Excélsior, México.

Zaragoza, J. M. (1982, 23 de julio). Se pedirá a la UNESCO aborde en su reunión la problemática de la Educación Física. La Afición, México, p. 17. 